\title{
Thermal-Vision Method of Investigations and Control of Device Based on Surface Plasmon Resonance
}

\author{
Gleb Dorozinsky, Vadim Dunaevsky, Vladimir Maslov* \\ V. Ye. Lashkaryov Institute of Semiconductor Physics of NAS of Ukraine, 45, prospekt Nauki, 03028, Kiev, Ukraine \\ *Corresponding author: vladmaslov@mail.ru
}

Copyright (C) 2013 Horizon Research Publishing All rights reserved.

\begin{abstract}
As is known, the miniaturization of analytical devices makes them more user-friendly and extends the sphere of their application. At the same time, however, the miniaturization deteriorates temperature schedules of the operation of devices because of localization that entails overheating and thereby making thermal errors of measurements more appreciable. This is especially critical to analytical devices operating on the basis of surface plasmon resonance because in that case a measurable value depends heavily on changes in a temperature. The main purpose of research is to use the method of nondestructive testing so as to analyze the thermal processes that take place in analytical devices operating on the basis of the surface plasmon resonance. This research suggests and shows the efficiency of the application of the thermal-vision method to detect the most heat-generating sources in analytical devices operating on the basis of the surface plasmon resonance. The results of this research can be used to improve the analytical devices as well as to streamline their design and fundamental basis.
\end{abstract}

Keywords thermal-vision nondestructive testing, plasmon resonance, thermal error of measurements

\section{Introduction}

Nondestructive testing of the quality of complex industrial goods is the essential part of the up-to-date production process. Methods of nondestructive testing more and more deeply penetrate into the sphere of maintenance, which allows to get information on the condition of a tested object, whereas this information cannot be obtained by means of other methods. The physical methods of nondestructive testing are often based on interaction of transmitting or reflected physical field with the tested object.

One of the most widely used methods for non-destructive testing are the thermal ones, whose operation is based on interaction of the thermal field inherent to the tested object with thermometric sensitive elements, that enables conversion of the field parameters into electric (or some other) signals and their transfer to a registering device. The thermal field of the tested object arises during operation of the latter and can disturb operating conditions that results in worsening of object's performance and its reliability.

Testing of the thermal processes, which take place in analytical devices, is of no small importance. Heat spreading in construction elements of analytical devices, which is caused by temperature gradients, leads to changes in linear dimensions of parts in a gear or sensitive elements in measuring channels. This makes appreciable errors in the results of measurements, which is especially critical to high-precision optical equipment.

In addition, heat released by units of an analytical device influences the measured object, changing its physical characteristics such as linear dimensions, electric conductivity, dielectric permittivity, the index of refraction, among other, that also distorts the results of measurements. Therefore, measurements of distribution and its changes for the thermal field released by units of analytical devices is a topical method to increase reliability and precision of measuring techniques.

Optical measurements on the basis of the phenomenon of surface plasmon resonance (SPR) are widely used for making a chemical and biological analysis based on registration of adsorption in gasiform or liquid mediums. The SPR is the optical stimulation of surface plasmons or waves of charge density at the interface between a conductor (for example, silver or gold) and dielectric (it may be gas, liquid or solid). The resonance wave vector, which is related to optical stimulation of surface plasmon waves, depends on the index of refraction of metal and dielectric contacting the former.

SPR occurs in a thin metal film with the negative dielectric permittivity (high electric conductivity), which is located on a transparent dielectric substrate.

The SPR phenomenon is observed as a sharp drop in the intensity of light reflected from the interface at some definite (resonance) angle of incidence. The fact is that conduction electrons of the thin metal film are stimulated by the laser at angles corresponding to a total internal reflection. Further, the fact that the resonance angle value depends on the concentration of analyzed substance contacting a sensitive 
element is important for designing of analytical devices. Gold is used as a sensitive layer more often than not, since it possesses high electric conductivity and chemical passivity.

The refractometric method based on the SPR phenomenon was successfully applied to analyze optical properties of a wide range of substances, namely gases used in medicine as anesthetics [1], liquids (for analyses of the binary system methanol - water [2]) and solids (inorganic solid particles [3] as well as Langmuir-Blodgett films[4]). Diagnostic devices based on SPR have high sensitivity to low concentrations of studied substances, which enables to use them not only as gas analyzers [5] but as precision analytical devices for lab investigations in food, chemical, pharmaceutical industries, agriculture, medicine and ecology [6-11].

The analysis of literary works and our own research shows that the enhancement of the exactness of measurements as well as reliability and efficiency of devices can be reached by the thermal stabilization of a measuring unit, increase in the number of measuring channels and improvement of the sensitive element. When taking these measurements, one of the problems is to reduce an error in the measurable value to a minimum.

The error value of measurement results is strongly influenced by temperature deviations [12-16] both in the measured object and in the whole measuring device. These temperature deviations are related to changes in an ambient temperature, the temperature of a measuring unit and, possibly, chemical processes in studied substances, which bring about the release or absorption of thermal power. In the course of measurements, this error may increase in consequence of the difference of temperatures at the start and the end of the measuring process.

As a result of investigations, in the works $[17,18]$ we suggested to exercise thermostatic control over not only the studied object but all the measuring equipment including containers with substances under test. This technique enables to reduce the temperature error in measurement results to a minimum and, in addition, to lessen a temperature load on measuring equipment, thus extending its service life. The thermostatic control enabled the fivefold enhancement of the exactness of measuring the concentration of studied substances and the determination of the character of running reactions (endothermic or exothermic) owing to a decrease in the temperature error.

To investigate biomolecular interactions, the methods, which are based on plasmon-polariton resonance, use measurements of the exact position of the plasmon resonance angle in devices with monochromatic beam $[19,20]$ or measurements of the wavelength corresponding to plasmon stimulation when a white light source is used [21, 22]. The application of optical methods for registering precise changes in the refractive index of a liquid medium and/or adsorbed molecular layers on a smooth surface is the worthwhile trend to develop fermentation and immune biosensors [23]. The device Biacore [24, 25] that ushered in an epoch of using the SPR phenomenon in biology has become widespread.
Its best advantage is the feasibility to register kinetics of biospecific reactions in situ. Previously, it required the introduction of special labels into reacting molecules. New methods of a fundamental and applied character, which are capable of registering the kinetics of the formation of biomolecular films on a metallic surface, are constantly evolved. These developments are represented by the following manufacturers: Biosuplar [26], Nippon Laser Electronics SPR [27], TISPR (USA) [28], Leica [29], SR7500DC [30, 31].

All the above devices contain very complex and expensive systems for thermostatic control. This is not always convenient to provide the necessary degree of thermal stability, which depends on the rate of endo- or exothermic chemical reactions in a measuring cell as well as on an ambient temperature. The miniaturization of devices makes them more user-friendly and extends the sphere of their application. However, the miniaturization worsens temperature schedules of the operation of devices because of localization that entails overheating and thereby making the temperature errors of measurement results more appreciable.

This means that a need to understand thermal management technique and a need for comprehensive data has never been urgent. Tools used in the past such as contact probes, thermocouples and fingers could not provide the thermal data quickly or ensure cost-effectiveness. Thanks to the availability of thermal imaging systems, today an engineer can make the requisite analysis timely without delaying product development schedules or interfering in production processes.

The purpose of the work was to detect thermal sources and study the process of spreading the thermal field in the analytical device, which operates on the basis of the SPR phenomenon, so as to make it more precise and reliable.

\section{Materials and Methods}

As an object for measurements, we chose the SPR spectrometer Plasmon-6 (Fig.1) developed at the V.E. Lashkaryov Institute of Semiconductor Physics under the National Academy of Sciences of Ukraine [32]. This device is capable of measuring one sample of the studied substance in an automated mode, displaying the obtained results on the monitor of a computer within 5-10 seconds. The optical scheme of the device is depicted on Fig. 1A. Its dimensions are $215 \times 130 \times 100 \mathrm{~mm}$.

As far as the design is concerned, the device is made as a mono-block structure consisting of the two bearing flat plates: the horizontal one and vertical rest, which are T-coupled with each other. The optical unit and measuring flow-cell with a prism are fixed on the one side of the rest, while the board of an electronic block for control and measurements as well as a step motor rotating the prism with a sensor are fixed on the other side of the rest. The optical unit and measuring flow-cell with a prism are depicted on Fig. 1B. The board of the electronic block for control and 
measurements as well as a step motor rotating the prism are depicted on Fig. 1C.

The rotation of the prism changes the angle of incidence for a monochromatic laser beam striking a prism surface that enables registration of a minimum on the SPR curve (Fig. 2).

To capture images, we used the thermal-vision camera Compix 320 [33]. This camera (Fig. 3) is a calibrated, radiometric, real-time thermal imaging camera. It requires low power and needs no cryogenic cooling. The 320 uses an amorphous silicon microbolometer detector and produces 320x240 pixel images. Combined with a personal computer (not included) and WinTES2 camera control software (included), the 320 provides a fast, easy image capture, analysis, and image storage. The 320 comes in either a standard enclosure or a smaller OEM housing. Accessory microscope lenses allow the Model 320 to reach pixel resolution of 6 microns.

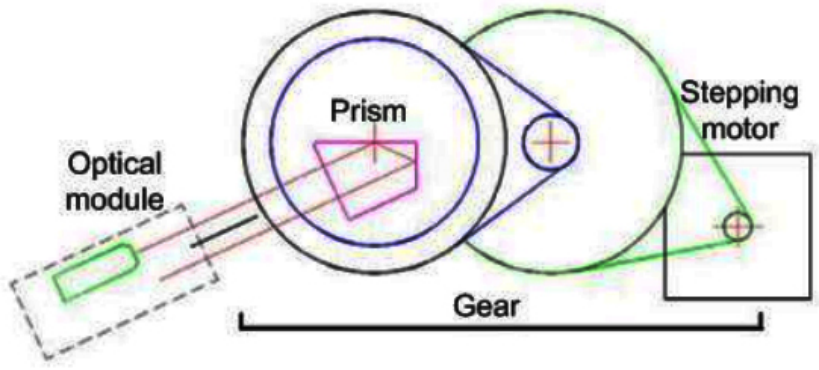

A

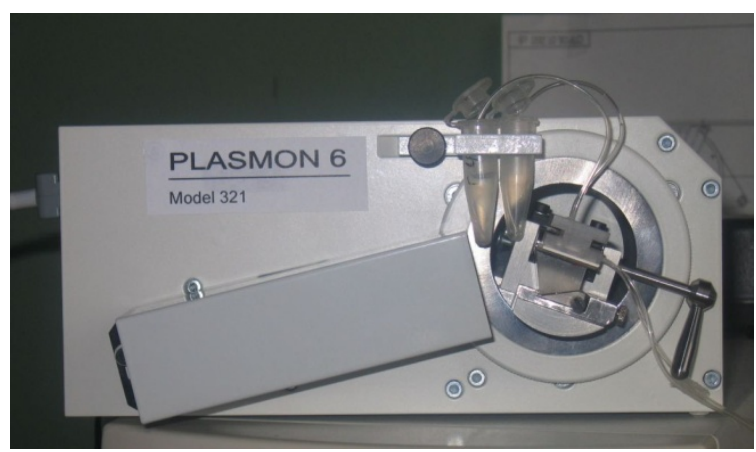

B

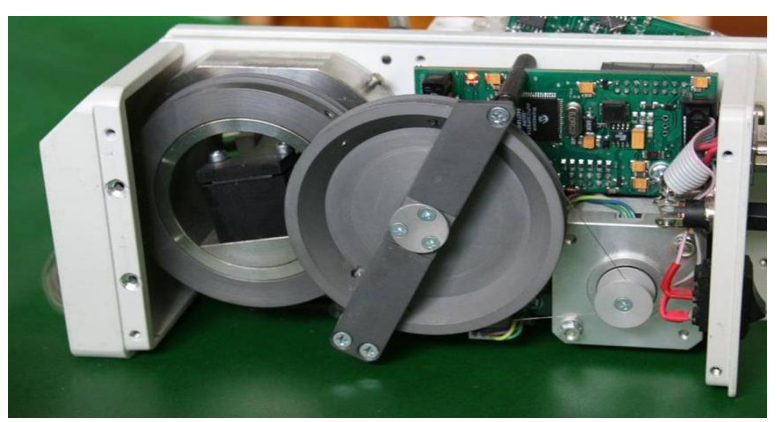

C

Figure 1. SPR spectrometer Plasmon-6: the kinematic scheme of the device (A); a view of the front panel of the device with the optical unit, prism and flow-cell (B); the reverse of the front panel on which the board of the electronic block, step motor and reduction system are fixed (C).

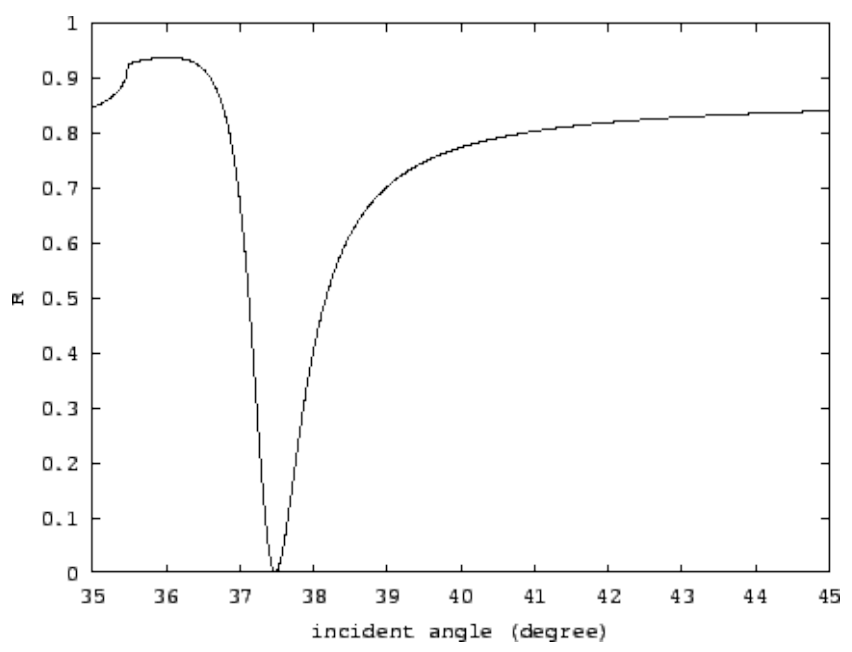

Figure 2. SPR curve

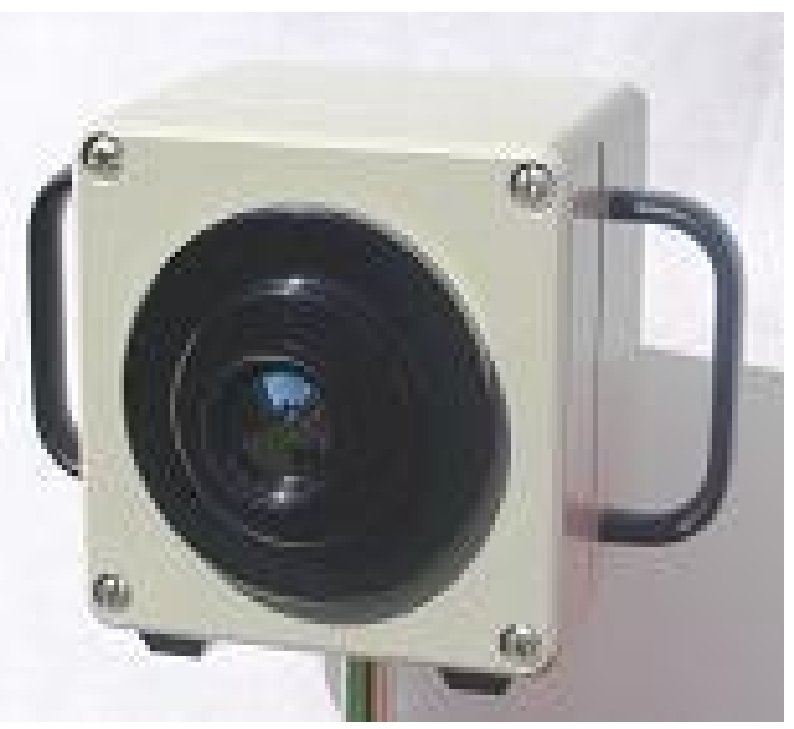

Figure 3. Thermal imaging camera Compix 320

The method for investigating and testing heat sources in the operating device was as follows:

1. Documenting the thermal image of the device in initial inoperative condition.

2. Documenting thermal images at several sequential intervals after the device has been turned on.

3. The duration of the investigating cycle totaled 40 minutes. Any increase in measurement duration did not result in getting of new thermal-vision information.

\section{Results}

In process of measurements, the ambient temperature within the effective area was $25 \pm 0.5{ }^{\circ} \mathrm{C}$ and the relative humidity made up $60 \%$. The documented thermal images are represented in Fig. 5-10. Fig. 5-8 illustrates temperature distribution over the front plane of the vertical plate where the optical unit and prism with the measuring flow-cell are located. Fig. 9 and 10 shows the temperature distribution 
over the back plane of the vertical plate where the board of the electronic control and measuring unit as well as the step motor are placed.

The thermal-vision camera fixed temperature values in the two points: the maximum temperature of the prism and that of the optical unit. Optical unit's temperature was measured when the housing was taken off (Fig. 4) that helped pinpoint the most heated source.

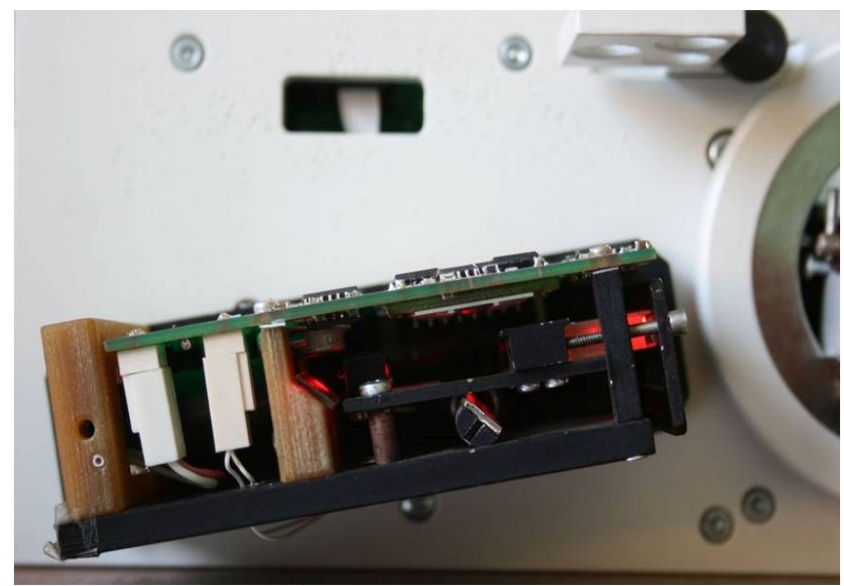

Figure 4. Optical unit of SPR spectrometer Plasmon-6

Fig. 5 shows the thermal image of the spectrometer Plasmon-6 when the power is off (initial condition). As is seen from the figure, the thermal field is of a uniform character. The temperature of the prism is equal to $26.76^{\circ} \mathrm{C}$.

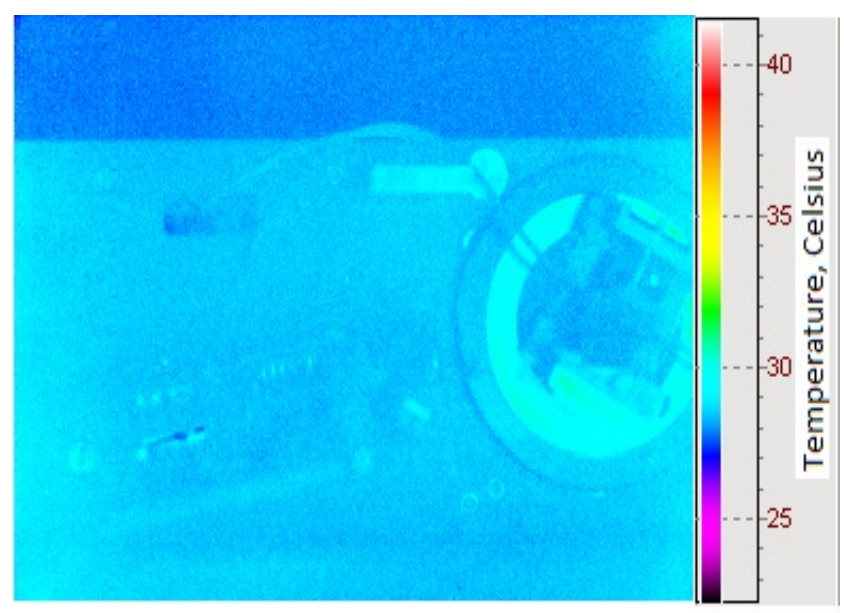

Figure 5. The thermal image of the front panel of the spectrometer Plasmon- 6 with the optical unit, prism and flow-cell. The device is turned off.

Fig. 6 shows the thermal image of the spectrometer Plasmon-6 after 10-minute operation when the power has been on. One can observe an increase in the temperature of the optical unit elements and prism. The temperature of the prism reached $27.65{ }^{\circ} \mathrm{C}$, while that of the optical unit amounted to $26.88^{\circ} \mathrm{C}$. Fig. 7 shows the thermal image of the switched-on device after 20-min. operation. The temperature of the prism and optical unit is $28.68{ }^{\circ} \mathrm{C}$ and $33.61^{\circ} \mathrm{C}$ respectively.

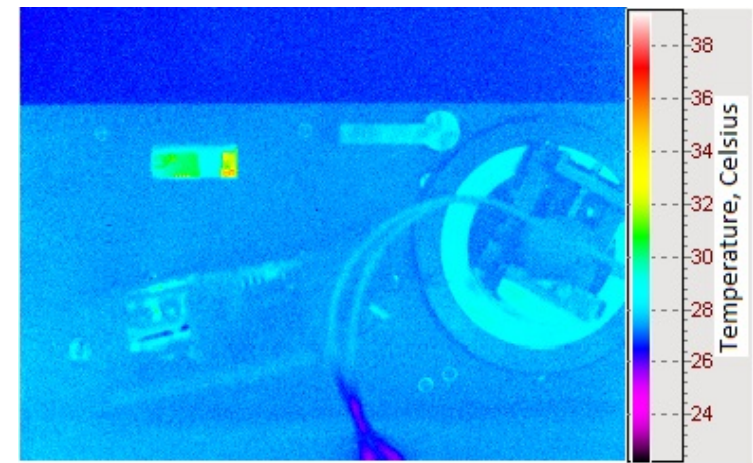

Figure 6. The thermal image of the front panel of the spectrometer Plasmon- 6 with the optical unit, prism and flow-cell. Device has been switched on after 10-min. operation.

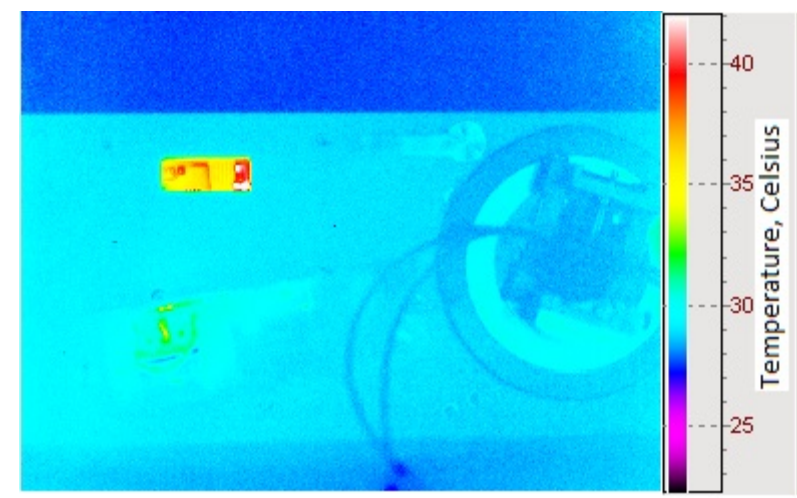

Figure 7. The thermal image of the front panel of the spectrometer Plasmon- 6 with the optical unit, prism and flow-cell. Device has been switched on after 20-min. operation.

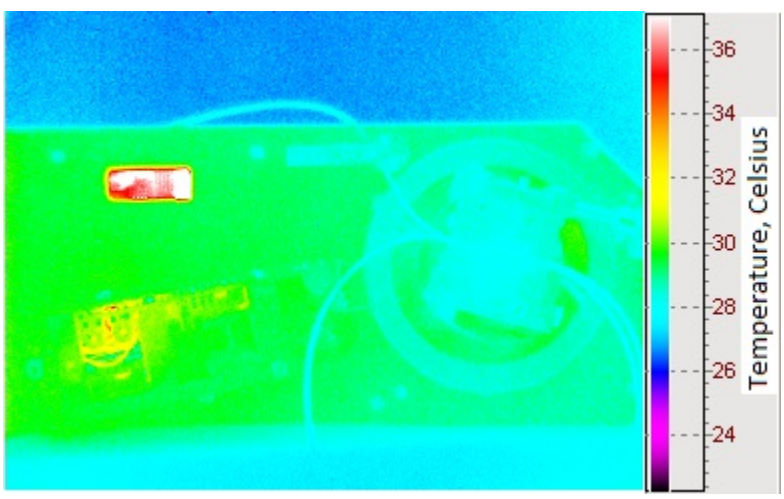

Figure 8. The thermal image of the front panel of the spectrometer Plasmon-6 with the optical unit, prism and flow-cell. Device has been switched on after 40-min. operation.

Fig. 8 shows the thermal image of the switched-on device after 40-min. operation. The temperature of the prism and optical unit is $28.2{ }^{\circ} \mathrm{C}$ and $34.32^{\circ} \mathrm{C}$ respectively. The observed decrease in prism's temperature is due to heat spreading over the construction elements near the prism.

The thermal image of the back plane of the vertical plate of the switched-on device after 20-min. operation is shown on Fig. 9. One can see that the electronic control unit is heated most of all. Fig. 10 shows the temperature distribution after 40-min. operation of Plasmon-6. It is clearly seen that 
heat spreads from the electronic board to other construction elements.

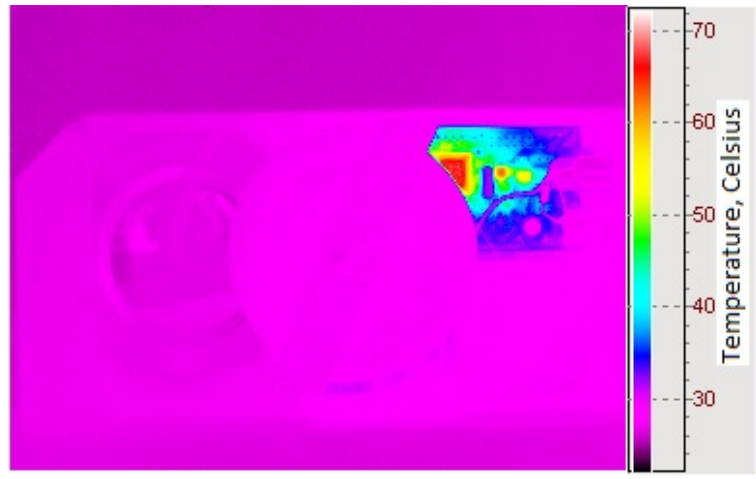

Figure 9. The thermal image of the reverse of the front panel of Plasmon-6, on which the board of the electronic control unit, step motor and reduction system are fixed. The image has been captured after 20 -min. operation of the device.

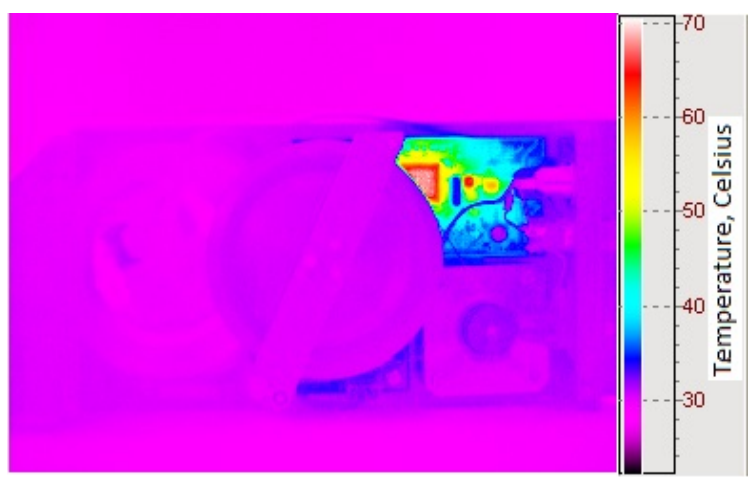

Figure 10. The thermal image of the reverse of the front panel of Plasmon-6, on which the board of the electronic control unit, step motor and reduction system are fixed. The image has been captured after 40-min. operation of the device.

\section{Discussion}

Our analysis of the obtained thermal images allowed us to find the following sources of heat inside the operating device:

1. The semiconductor laser in the optical unit. Its temperature has increased from $26{ }^{0} \mathrm{C}$ to $35^{\circ} \mathrm{C}$ over the course of 40-min. operation.

2. The main heat source in the electronic unit is the control processor chip.

3. As is evident from measurements, the step motor is not the heat-affected source. Fig. 10 shows that heat is spreading from the board of the control unit to other components.

\section{Conclusion}

The results of thermal-vision thermography enabled us to analyze the working principle of the device Plasmon- 6 and find main heat sources inside it. This allows us to give the following directions to enhance precision of measurements with the help of Plasmon- 6 for the purpose of lowering the influence of a temperature:

1. Precise measurements should be taken during the first minutes of operation of the device. Then, the device should be turned off, and repeated measurements should be taken only after a temperature has become steady.

2. It is necessary to equip the device with a natural cooling or air conditioning system for the processor chip and semiconductor laser as well as to perforate the case for the purpose of facilitating heat removal.

\section{REFERENCES}

[1] B. Liedberg, C. Nylander, I. Lundstrom, Surface Plasmon Resonance For Gas Detection and Biosensing, Sensors and Actuators, № 4, 299-304, 1983.

[2] K. Matsubara, S. Kawata, S. Minami, Optical Chemical Sensor Based on Surface Plasmon Measurement, Applied Optics, № 27, 1160-1163, 1988

[3] H. Xu and M. Kall, Modeling the optical response of nanoparticle-based surface plasmon resonance sensors, Sensors and Actuators B: Chemical, Vol. 87, № 2, 244-249, 2002.

[4] R. Rella, P. Siciliano, F. Quaranta, T. Primo, L. Valli, L. Schenetti, A. Mucci, D. Iarossi, Gas Sensing Measurements and Analysis of the Optical Properties of Poly [3-(butylthio)thiophene] Langmuir-Blodgett films Sensors and Actuators B68, 203-209, 2000.

[5] A.V. Samoylov, V.M. Mirsky, Q. Hao, C. Swart, Y.M. Shirshov, O.S.Wolfbeis, Nanometer-thick SPR sensor for gaseous $\mathrm{HCl}$, Sensors and Actuators B Chem, Vol.106, $369-372,2005$.

[6] N. Gridina, G. Dorozinsky, R. Khristosenko, V. Maslov, A. Samoylov, Yu. Ushenin, Yu. Shirshov, Surface plasmon resonance biosensor, Sensors \& Transducers Journal. Vol.149, № 2, 60-68, 2013.

[7] A. V. Samoylov, Yu. V. Ushenin, R. V. Khristosenko, Application of surface plasmon resonance spectrometer for determination of optical parameters of the thin polyaniline films, "International Conference «Functional Materials» ICFM, 2007.

[8] N.Ya. Gridina, A.V. Samoilov, Yu.V. Ushenin, Yu.M. Shirshov, Diagnostic possibility demonstration' of cerebrum oncologic diseases by biosensor based on surface plasmon resonance, International Conference "Functional Material” ICFM-2009, Partenit, Ukraine, 2009.

[9] Yu.M. Shirshov, A.V. Samoylov, S.A. Zinyo, E.R. Surovceva, V. Mirskiy, Bimetallic Layers Increase Sensitivity of Affinity Sensors Based on Surface Plasmon Resonance, Sensors, Vol.2, 62-70, 2002.

[10] K.V. Kostyukevych, R.V. Khristosenko, Yu.M. Shirshov, S.A. Kostyukevych, A.V. Samoylov, V.I. Kalchenko Multi-element gas sensor based on surface plasmon resonance: recognition of alcohols by using calixarene films, Semiconductor Physics, Quantum Electronics and 
Optoelectronics, V.14, №3, 313-320, 2011.

[11] A. Rachkov, Yu. Holodova, Yu. Ushenin, D. Miroshnichenko, G. Telegeev, A. Soldatkin, Development of Bioselective Element of SPR Spectrometer for Monitoring of Oligonucleotide Interactions and Comparison with Thermodynamic Calculations, Sensor Letters, Vol. 7, 2009.

[12] C. S. Moreira et al., Temperature-dependent sensitivity of surface plasmon resonance sensors at the gold-water interface, Sensors and Actuators B, № 134, 854-862, 2008.

[13] H. P. Chiang et al., Surface plasmon resonance monitoring of temperature via phase measurement, Optics Communications, № 241, 409-418, 2004.

[14] Özdemir S. K. Temperature Effects on Surface Plasmon Resonance: Design Considerations for an Optical Temperature Sensor, Journal of light wave technology, V.21, № 3, 805-815, 2003.

[15] Lin Kai-Qun et al., Temperature Effects on Prism-Based Surface Plasmon Resonance Sensor, Chin.Phys.Lett., V. 24, №11, 3081-3084, 2007.

[16] K Lin et al., Numerical and experimental investigation of temperature effects on the surface plasmon resonance sensor, Chinese Optics Letters, V.7, № 5, 428-431, 2009.

[17] G. Dorozinsky, V. Maslov, A. Samoylov, Yu. Ushenin. Reducing measurement uncertainty of instruments based on the phenomenon of surface plasmon resonance, 21st International Congress on Photonics in Europe Collocated with LASER 2013 World of PHOTONICS. Optical Metrology, Munich: Germany, 2013.

[18] G. Dorozinsky, V. Maslov, A. Samoylov, Yu. Ushenin. Reducing measurement uncertainty of instruments based on the phenomenon of surface plasmon resonance, American Journal of Optics and Photonics, Vol.1, № 3, 17-22, 2013.

[19] U. Jonsson, L. Fagerstam, B. Ivarsson Real-time biospecific interaction analysis using surface plasmon resonance and a sensor chip technology, BioTechniques, Vol.11, 620-627, 1991.

[20] R.G. Woodbury, C. Wendin, J. Clendenning, J. Mendelez, J. Elkind, D. Bartholomew, S. Brown, C. Furlong, Construction of biosensors using a gold-binding polypeptide and a miniatureintegrated surface plasmon resonance sensor, Biosensors and Bioelectronics, Vol.13, 1117-1126, 1998.

[21] J. Homola, The sensitivity of surface plasmon resonance sensors with spectral interrogation, Sensors and Actuators, B., Vol.41, 207-211, 1997.

[22] H.P. Ho, S.Y. Wu, M. Yang, A.C. Cheung Application of white light-emitting diode to surface plasmon resonance sensors, Sensors and Actuators, B., Vol.80, 89-94, 2001.

[23] W. Huber et al. Direct optical immunosensing, Sensors and Actuators, B. Vol.6, 122-126, 1992.

[24] B. Liedberg, I. Lundstrom, E. Stenberg, Principles of Biosensing with an Extended Coupling Matrix and Surface Plasmon Resonance, Sensors and Actuators B11, 63-72, 1993.

[25] www.biacore.com

[26] www.micro-analytical systems.de

[27] www.nle-lab.co.jp

[28] www.ti.com/spr

[29] www.leica-ead.com

[30] www.reichert.com

[31] www.xantec.com

[32] www.plasmon.org.ua

[33] www.compix.com 